\title{
A Predictive Model, Using Data Mining Approach for Clinical Decision-making: Value of Laboratory Tests in COVID-19 Hospitalized Patients
}

\author{
Atefeh Mousavi ${ }^{1}$, Soheila Rezaei ${ }^{1}$, Jamshid Salamzadeh ${ }^{2}$, Ali Mirzazadeh ${ }^{3}$, Farzad Peiravian ${ }^{5}$ and Nazila Yousefi ${ }^{5,}$ \\ ${ }^{1}$ PhD Candidate, Department of Pharmacoeconomics and Pharma Management, School of Pharmacy, Shahid Beheshti University of Medical Sciences, Tehran, Iran \\ 2 Professor, Department of Clinical Pharmacy, School of Pharmacy, Shahid Beheshti University of Medical Sciences, Tehran, Iran \\ ${ }^{3}$ Assistant Professor, Department of Epidemiology and Biostatistics, School of Medicine, University of California, San Francisco, California, USA \\ ${ }_{4}^{4}$ Assistant Professor, Department of Pharmacoeconomics and Pharma Management, School of Pharmacy, Shahid Beheshti University of Medical Sciences, \\ Tehran, Iran
}

* Corresponding author: Nazila Yousefi, Department of Pharmacoeconomics and Pharma Management, School of Pharmacy, Shahid Beheshti University of Medical Sciences, Tehran, Iran. Tel: +982188665692; Email: n.yousefi@sbmu.ac.ir

Received 2021 February 05; Revised 2021 February 24; Accepted 2021 April 19.

\begin{abstract}
Background: Reports, mostly from high-income countries, have shown a wide range of symptoms, clinical profiles, and outcomes for patients diagnosed with COVID-19. However, little is known about these issues in developing countries.

Objectives: This research used medical records in 15 hospitals in Tehran, the capital city of Iran, to assess predictors of in-hospital mortality in patients diagnosed with COVID-19.

Methods: The required information was extracted from patients' medical records, including age, gender, laboratory data (complete blood count, serum electrolytes, and liver, renal, and muscle injury tests) at admission, and the outcome of in-hospital mortality (yes/no) of 4,542 adult patients with confirmed COVID-19. This research used logistic regression to assess the predictors for mortality (measured as adjusted odds ratio [aOR]) and Chi-square automatic interaction detector to classify high-risk patients in different age groups as a decision tree model. Two models were developed through a machine learning approach.

Results: Overall, 822 (18.09\%) cases passed away in the hospital. Mortality risk was increased from 4.33\% in patients aged 18-40 years old to $40.96 \%$ in those aged $80+$ years old. After adjusting for covariates, age (aOR 1.62 to 7.05 vs. those aged 18-40 years old), high aspartate transaminase (aOR 1.64 to 3.21), high alkaline phosphatase (aOR=2.17), low sodium (aOR=1.31), high sodium level (aOR=5.05), high potassium $(\mathrm{aOR}=2.41)$, low calcium $(\mathrm{aOR}=2.31)$, high creatine phosphokinase $(\mathrm{aOR}=2.21$ to 2.24$)$, and high creatinine $(\mathrm{aOR}=3.43)$ were significantly associated with in-hospital mortality.

Conclusion: Based on the results of our study, the mortality rate was high among in-hospital patients, particularly among older age and those who had liver and renal dysfunctions, muscle injury, and electrolyte imbalance at admission. Triage and special care for these highrisk patients can improve in-hospital outcomes.
\end{abstract}

Keywords: COVID-19, Decision tree model, Laboratory test, Machine learning, Odds ratio, Risk factor

\section{Background}

In December 2019, an outbreak of coronavirus disease 2019 (COVID-19) began in Wuhan, China, and has continued to spread globally (1). Coronavirus disease 2019 can cause fever, cough, fatigue, diarrhea, shortness of breath, and high mortality due to severe respiratory symptoms $(2,3)$. The first official announcement of deaths caused by COVID-19 in Iran was made on Feb 19, 2020 (4). Previous experiences with other outbreaks, such as Middle East Respiratory Syndrome and pandemic influenza, have shown that the identification of high-risk patients for hospitalization can help healthcare providers and emergency staff find patients who would benefit the most from early, available treatments. In addition, policymakers can use this information to forecast healthcare needs (5).

The machine learning approach, which can develop more powerful models, such as logistic regression and decision tree models, has been frequently used in various healthcare applications, including the prediction of disease risk factors and mortality rate (MR) and classification and identification of high-risk patients $(6,7)$. Decision analysis models, such as Chi-square automatic interaction detector (CHAID), which is used in this study, are quantitative methods to identify strategies that can affect outcomes in the healthcare system under uncertain conditions (8).

In this study, a two-stage method was used to measure the risk of mortality among the hospitalized patients and the possible association between demographic and clinical characteristics and mortality risk. In this regard, logistic regression, followed by a decision tree (i.e., CHAID) analysis, was applied to identify COVID-19 risk factors and detect the high-risk patients in different age groups, respectively.

\section{Objectives}

This research used medical records in 15 hospitals in Tehran, the capital city of Iran, to assess predictors of in-hospital mortality in patients diagnosed with COVID-19. 


\section{Methods}

\subsection{Study design}

This retrospective cohort study was conducted on 4,542 patients with severe symptoms of COVID-19 hospitalized in 15 hospitals in Tehran, Iran. These patients were later divided into two groups of recovered and discharged patients and deceased patients. The predictors were demographic characteristics of gender and age, along with different laboratory tests. Moreover, oxygen therapy and mechanical ventilation were set as intervention variables. All data analyses were performed using the IBM SPSS Modeler software (version 18.0). The SPSS Modeler performed data preparation before primary analysis by replacing null values with the mean for continuous fields followed by z-transformation rescaling. It increases the performance and accuracy of the model through machine learning and artificial intelligence techniques (9).

The data were then separated into two sections, namely training $(80 \%)$ and testing $(20 \%)$, which provided an infrastructure for the next steps. Due to a large number of model predictors, the use of the training and testing dataset helps to track overfitting, which is conducted based on the fitness criteria of the testing dataset. Additionally, before the principal analysis, a feature selection or variable selection was performed to choose the most relevant model predictors (7). Afterward, multivariable and univariable logistic regressions were executed in three steps to identify the risk factors of COVID-19. In the final stage of the analysis, a decision model was developed to identify high-risk patients.

\subsection{Data collection}

The statistical population of this study consisted of 4,791 adults with a definitive diagnosis of COVID19 admitted to 15 university hospitals in Tehran, Iran, from 20 February to 18 April, 2020. However, 249 patients were excluded from our analysis due to missing data on their laboratory tests. Their data was routinely registered on the Hospital Information System and centralized access to them was made possible through the Intelligent Hospital Management System. The patients' clinical records included fever (above $38^{\circ} \mathrm{C}$ ), shortness of breath, hypoxia, chest pain, severe cough, and loss of consciousness. Patients were divided into two groups of recovered and discharged patients and deceased patients. The definitive diagnosis of COVID-19 was made according to the Iranian Ministry of Health protocol using throat-swab specimens and the reverse transcription polymerase chain reaction test. Available data included age, gender, inpatient wards, supportive interventions of oxygen therapy and mechanical ventilation, duration of hospitalization, and laboratory test results on admission. These laboratory tests were:
1) Complete blood count tests, including red blood cells (RBC) count, white blood cells count, hemoglobin, hematocrit, platelets count, mean corpuscular volume, mean corpuscular hemoglobin $(\mathrm{MCH})$, mean corpuscular hemoglobin concentration, red cell distribution width, mean platelet volume, lymphocytes and neutrophils count, and MIX (the ratio of eosinophil, basophil, and monocyte to total white blood cells).

2) Liver function enzymes, including alanine transaminase (ALT), aspartate transaminase (AST), alkaline phosphatase (ALP).

3) Heart and muscle injury enzymes, including troponin, creatine kinase myocardial band, creatine phosphokinase (CPK).

4) Other tests, including creatinine, uric acid, serum $\mathrm{Ca}$, serum $\mathrm{K}$, serum $\mathrm{Na}$, 25-hydroxyvitamin $\mathrm{D}$, fasting blood sugar, D-Dimer, bicarbonate, blood PH, base excess, and c-reactive protein (CRP).

\subsection{Statistics}

Three main steps were taken to perform data analysis. These steps were as follows:

\subsubsection{STEP 1) A multivariable logistic regression analysis to identify the significant risk factors of COVID-19}

A multivariable logistic regression (MLR) analysis was performed to predict any relationship between predictors and the survival outcome (recovered or deceased).

\subsubsection{STEP 2) Univariate and multivariate logistic} regression analyses to calculate the crude and adjusted odds ratio of the identified risk factors

The predictors having a significant association $(\mathrm{P}<0.05)$ with the MLR outcome from step 1 were selected and transformed into a set of appropriate categorical variables. This step is performed to identify the relationship between different levels (normal or abnormal levels) of laboratory tests and MR due to COVID-19 and calculate the odds ratio (OR) by logistic regression. Accordingly, some of the laboratory tests were divided into two or three categories (i.e., normal range and above and below the normal range) and some were divided into more than three categories for their extra-outlier values. Subsequently, the predictors were entered into univariate logistic regression (ULR)one by one to calculate the crude $O R$, and once more all the predictors were entered into the MLR model together to obtain adjusted OR.

\subsubsection{STEP 3) A decision tree model to identify high- risk patients}

The identified predictors from steps one and two were incorporated into the CHAID model to develop a decision tree model and identify the high-risk patients. 


\section{Results}

\subsection{Descriptive Statistics}

The included patients had severe symptoms, such as fever higher than $38^{\circ} \mathrm{C}$, severe cough, shortness of breath, respiratory rate of $>30$ times/min, hypoxia (p02<93\%), chest pain, and loss of consciousness. The criteria of discharge from the hospital were lacking a fever and need for an antipyretic drug for
$72 \mathrm{~h}, \mathrm{p0} 2$ of $>93 \%$, and improvement in the clinical and respiratory symptoms. The mean scores of hospitalization length for the survived and deceased groups were obtained at $4.76 \pm 4.77$ and $6.45 \pm 7.21$ days, respectively (Table 1). The treatment program was identical in all hospitals and in accordance with the COVD-19 treatment guidelines proposed by the Iran Ministry of Health, unless concurrent diseases required specific treatments.

\begin{tabular}{|c|c|c|c|c|}
\hline & $\begin{array}{c}\text { Total } \\
\text { N (Percent) }\end{array}$ & $\begin{array}{c}\text { Survived } \\
\mathrm{N} \text { (Percent) }\end{array}$ & $\begin{array}{c}\text { Deceased } \\
\text { N (Percent) }\end{array}$ & P-value \\
\hline \multicolumn{5}{|l|}{ Demographic characteristics } \\
\hline Gender & $2,676(58.92 \%)$ & $2,169(47.76 \%)$ & 507 (11.15\%) & 0.00 \\
\hline Male & $1,866(41.08 \%)$ & $1,551(34.14 \%)$ & $315(6.93 \%)$ & 0.00 \\
\hline Female & $57.94 \pm 17.27$ & $55.55 \pm 16.84$ & $68.72 \pm 14.93$ & 0.00 \\
\hline Age (Mean \pm SD) & $57.94=17.28$ & & & \\
\hline Age groups & $785(17.28 \%)$ & $723(15.91 \%)$ & $34(0.74 \%)$ & \\
\hline $\begin{array}{l}18-40 \\
40-50\end{array}$ & $705(15.52 \%)$ & $671(14.77 \%)$ & $59(1.29 \%)$ & \\
\hline $\begin{array}{l}40-50 \\
50-60\end{array}$ & $885(19.48 \%)$ & $767(16.88 \%)$ & $118(2.59 \%)$ & \\
\hline $\begin{array}{l}50-60 \\
60-70\end{array}$ & $843(18.56 \%)$ & $689(15.17 \%)$ & $154(3.39 \%)$ & \\
\hline $\begin{array}{r}60-10 \\
70-80\end{array}$ & $760(16.73 \%)$ & $535(11.78 \%)$ & $225(4.95 \%)$ & \\
\hline $70-80$ & $564(12.42 \%)$ & $333(7.33 \%)$ & $231(5.09 \%)$ & \\
\hline Above 80 & $1,029(22.65 \%)$ & $517(11.38 \%)$ & $512(11.27 \%)$ & 0.00 \\
\hline Admitted to ICU/CCU & $3,513(77.34 \%)$ & $3,203(70.51 \%)$ & $310(68.24 \%)$ & 0.00 \\
\hline $\begin{array}{l}\text { Went on a ventilator } \\
\text { Duration of hospitalization (days) (Mean } \pm \text { SD) }\end{array}$ & $5.10 \pm 5.33$ & $4.76 \pm 4.77$ & $6.45 \pm 7.21$ & 0.00 \\
\hline \multicolumn{5}{|l|}{ Clinical characteristic } \\
\hline \multicolumn{5}{|l|}{ Complete blood count } \\
\hline Abnormal MCV & $60(11.56 \%)$ & $42(8.09 \%)$ & $18(3.46 \%)$ & 0.45 \\
\hline Abnormal MCH & $80(15.41 \%)$ & $52(10.02 \%)$ & $28(5.39 \%)$ & 0.00 \\
\hline Abnormal MCHC & $227(43.07 \%)$ & $175(29.79 \%)$ & $52(9.86 \%)$ & 0.29 \\
\hline Abnormal MIX & $265(50.28 \%)$ & $202(38.32 \%)$ & $63(11.95 \%)$ & 0.06 \\
\hline Abnormal WBC & $181(34.34 \%)$ & $145(27.51 \%)$ & $36(6.83 \%)$ & 0.14 \\
\hline Abnormal RBC & $144(27.32 \%)$ & $109(20.68 \%)$ & $35(6.64 \%)$ & 0.08 \\
\hline Abnormal Platelets & $149(28.71 \%)$ & $102(19.65 \%)$ & $47(9.03 \%)$ & 0.03 \\
\hline Abnormal Hemoglobin & $211(40.42 \%)$ & $168(32.18 \%)$ & $43(8.24 \%)$ & 0.63 \\
\hline Abnormal Hematocrit & $424(81.70 \%)$ & $310(59.73 \%)$ & $114(21.97 \%)$ & 0.03 \\
\hline Abnormal RDW-CV & $159(30.69 \%)$ & $123(23.74 \%)$ & $36(6.95 \%)$ & 0.21 \\
\hline Abnormal PDW & $49(9.98 \%)$ & $32(6.52 \%)$ & $17(3.46 \%)$ & 0.02 \\
\hline Abnormal MPV & $21(4.28 \%)$ & $16(3.26 \%)$ & $5(10.2 \%)$ & 0.44 \\
\hline Abnormal Neutrophils & $508(88.35 \%)$ & $397(69.04 \%)$ & $111(19.30 \%)$ & 0.01 \\
\hline Abnormal Lymphocyte & $412(69.01 \%)$ & $327(54.77 \%)$ & $85(14.24 \%)$ & 0.50 \\
\hline \multicolumn{5}{|l|}{ Abnormal liver function tests } \\
\hline Abnormal AST & $1,945(60.20 \%)$ & $1,561(48.32 \%)$ & $284(8.79 \%)$ & 0.00 \\
\hline Abnormal ALT & $592(19.67 \%)$ & $486(16.15 \%)$ & $106(3.52 \%)$ & 0.40 \\
\hline Abnormal ALP & $2,391(82.05 \%)$ & $1,936(66.43 \%)$ & $455(15.61 \%)$ & 0.00 \\
\hline \multicolumn{5}{|l|}{ Muscles injury-related enzymes } \\
\hline Abnormal CPK & $824(25.94 \%)$ & $643(20.24 \%)$ & $181(5.70 \%)$ & 0.00 \\
\hline Abnormal CKMB & $257(16.40 \%)$ & $208(13.27 \%)$ & $49(3.13 \%)$ & 0.21 \\
\hline Abnormal Troponin & $206(13.92 \%)$ & $155(10.47 \%)$ & $51(3.45 \%)$ & 0.00 \\
\hline \multicolumn{5}{|l|}{ Other test results } \\
\hline Abnormal Vitamin D3 & $157(71.36 \%)$ & $111(50.45 \%)$ & $46(20.91 \%)$ & 0.00 \\
\hline Abnormal Serum Ca & $874(34.34 \%)$ & $679(26.68 \%)$ & $195(7.66 \%)$ & 0.00 \\
\hline Abnormal Serum K & $343(7.76 \%)$ & $282(6.38 \%)$ & $61(13.8 \%)$ & 0.56 \\
\hline Abnormal Serum Na & $813(18.38 \%)$ & $605(13.68 \%)$ & $208(4.70 \%)$ & 0.00 \\
\hline Abnormal D-Dimer & $270(57.94 \%)$ & $150(32.18 \%)$ & $120(25.75 \%)$ & 0.00 \\
\hline Abnormal CRP & $2055(85.45 \%)$ & $1,611(66.98 \%)$ & $444(18.46 \%)$ & 0.00 \\
\hline Abnormal Creatinine & $884(19.60 \%)$ & $546(12.10 \%)$ & $338(7.49 \%)$ & 0.00 \\
\hline Abnormal FBS & $851(75.71 \%)$ & $683(60.76 \%)$ & $168(14.94 \%)$ & 0.01 \\
\hline Abnormal Uric Acid & $255(44.04 \%)$ & $178(30.74 \%)$ & $77(13.30 \%)$ & 0.74 \\
\hline Abnormal Blood PH & $237(42.47 \%)$ & $176(31.54 \%)$ & $61(10.93 \%)$ & 0.03 \\
\hline Abnormal $\mathrm{HCO}_{3}$ & $327(58.60 \%)$ & $260(46.59 \%)$ & $67(12.01 \%)$ & 0.07 \\
\hline Abnormal BE & $225(63.20 \%)$ & $180(50.56 \%)$ & $45(12.64 \%)$ & 0.02 \\
\hline
\end{tabular}

ICU/CCU: Intensive care unit/critical care unit; MCV: Mean corpuscular volume; MCH: Mean corpuscular hemoglobin; MCHC: Mean corpuscular hemoglobin concentration; MIX: Ratio of eosinophil, basophil, and monocyte to total white blood cells; WBC: White blood cell; RBC: Red blood cell; RDW-CV: Red cell distribution width-coefficient of variation; PDW: Platelet distribution width; MPV: Mean platelet volume; AST: Aspartate transaminase; ALT: Alanine transaminase; ALP: Alkaline phosphatase; CPK: Creatine phosphokinase; CKMB: Creatine kinase myocardial band; CRP: C-reactive protein; FBS: Fasting blood sugar; $\mathrm{HCO}_{3}$ : Bicarbonate; BE: Base excess 
Among all the hospitalized patients $(n=4,542)$, who were in the age range of 18-97 years and had severe symptoms, 822 (18.09\%) and 3,720 (81.90\%) cases passed away during hospitalization and recovered and discharged, respectively. The mean and median [interquartile range] age scores of patients were, respectively, $55.55 \pm 16.84$ and 56.00 [27.00] for the survived group, and 68.72 \pm 14.93 and 71.00 [21.25] for the deceased one. Furthermore, the majority of patients were male (58.92\%). Some patients had abnormal laboratory test results, which are presented in Table 1.

Compared to those who survived, patients who passed away in the hospital were significantly older with abnormal laboratory tests, such as $\mathrm{MCH}$, platelets, hematocrit, serum electrolytes, and liver enzymes (Table 1).

As mentioned in the method section, before entering all the independent variables in the MLR model, a feature selection algorithm was used to define the most important predictors associated with the target variable. According to the feature selection results, apart from RBC, platelets, hemoglobin, hematocrit, and platelet distribution width, all laboratory tests, age, gender, and the need for special care, such as oxygen therapy and ventilation for survived patients, were entered in the model as potential variables correlated with disease prognosis (Table 2).

\begin{tabular}{|c|c|c|c|c|c|}
\hline Lab test & $\begin{array}{c}\text { Total } \\
(n=4,542) \text { Mean } \pm \text { SD }\end{array}$ & $\begin{array}{c}\text { Died } \\
(n=822) \text { Mean } \pm S D\end{array}$ & $\begin{array}{c}\text { Survived } \\
(n=3720) \text { Mean } \pm S D\end{array}$ & Feature sel & ction results \\
\hline Predictors & Target groups & Mean & SD & $\begin{array}{c}\text { Value of } \\
\text { importance }\end{array}$ & Importance \\
\hline Age & $(4,542) 57.94 \pm 17.27$ & (822) $8.72 \pm 14.92$ & $(3,720) 55.55 \pm 14.92$ & 1.00 & Important \\
\hline Gender & $(4,210) 1.59 \pm 0.49$ & $(760) 1.62 \pm 0.48$ & $(3,450) 1.58 \pm 0.00$ & 0.92 & Marginal \\
\hline \multicolumn{6}{|c|}{ Complete blood count } \\
\hline MCV & (519) $86.43 \pm 6.38$ & (145) 87.14 14.42 & (374) $86.15 \pm 5.91$ & 1.00 & Important \\
\hline $\mathrm{MCH}$ & (519) $31.30 \pm 1.90$ & (145) $31.02 \pm 2.20$ & (374) $31.40 \pm 1.75$ & 1.00 & Important \\
\hline MCHC & (519) $33.56 \pm 1.45$ & (145) $33.13 \pm 1.60$ & (374) $33.73 \pm 1.35$ & 1.00 & Important \\
\hline MIX & (519) $6.06 \pm 3.05$ & $(145) 4.97 \pm 2.66$ & (374) $6.49 \pm 3.10$ & 1.00 & Important \\
\hline WBC & (519) $8.49 \pm 5.02$ & (145) $11.34 \pm 6.52$ & (374) $7.39 \pm 3.77$ & 1.00 & Important \\
\hline $\mathrm{RBC}$ & (519) $4.24 \pm 0.69$ & (145) $4.19 \pm 0.85$ & (374) $4.26 \pm 0.63$ & 0.85 & Unimportant \\
\hline Platelets & (519) $204.78 \pm 82.32$ & $(145) 96.97 \pm 82.38$ & (374) $207.82 \pm 82.20$ & 0.75 & Unimportant \\
\hline Hemoglobin & (519) $12.26 \pm 2.01$ & $(145) 12.06 \pm 2.46$ & (374) $12.33 \pm 1.80$ & 0.47 & Unimportant \\
\hline Hematocrit & (519) $36.47 \pm 5.56$ & (145) $36.33 \pm 6.91$ & (374) $36.53 \pm 4.94$ & 0.18 & Unimportant \\
\hline RDW-CV & (515) $14.40 \pm 2.52$ & (143) $15.14 \pm 2.35$ & (372) $14.11 \pm 2.52$ & 1.00 & Important \\
\hline PDW & (491) $13.50 \pm 2.47$ & (135) $13.40 \pm 2.63$ & (356) $13.53 \pm 2.41$ & 0.76 & Unimportant \\
\hline MPV & (491) $9.45 \pm 1.10$ & (135) $9.78 \pm 1.06$ & (356) $9.32 \pm 1.09$ & 1.00 & Important \\
\hline Neutrophils & (567) $75.12 \pm 11.40$ & (143) $82.64 \pm 9.08$ & (424) $72.58 \pm 10.98$ & 1.00 & Important \\
\hline Lymphocyte & (581) $18.68 \pm 10.59$ & (148) $11.84 \pm 8.74$ & (433) $21.02 \pm 10.15$ & 1.00 & Important \\
\hline \multicolumn{6}{|l|}{ Liver function tests } \\
\hline AST & $(3,216) 77.36 \pm 110.28$ & (679) $26.32 \pm 188.05$ & $(2,537) 64.26 \pm 71.75$ & 1.00 & Important \\
\hline ALT & $(2,989) 48.63 \pm 97.26$ & (638) $73.28 \pm 155.15$ & $(2,351) 41.94 \pm 72.75$ & 1.00 & Important \\
\hline ALP & $(2,189) 07.09 \pm 152.00$ & (433) $48.74 \pm 189.23$ & $(1,756) 196.83 \pm 139.49$ & 1.00 & Important \\
\hline \multicolumn{6}{|c|}{ Muscles injury-related enzymes } \\
\hline $\mathrm{CPK}$ & $(3,152) 294.31 \pm 708.86$ & (664) 500.33 \pm 1099.24 & $(2,488) 239.33 \pm 547.87$ & 1.00 & Important \\
\hline CKMB & $(1,615) 27.90 \pm 40.18$ & (318) $45.61 \pm 62.64$ & $(1,297) 23.56 \pm 30.91$ & 1.00 & Important \\
\hline Troponin & $(1,475) 0.99 \pm 7.00$ & (316) $2.33 \pm 12.30$ & $(1,159) 0.62 \pm 4.54$ & 1.00 & Important \\
\hline \multicolumn{6}{|l|}{ Serum electrolytes } \\
\hline Serum Ca & $(2,529) 8.78 \pm 0.85$ & $(615) 8.56 \pm 0.97$ & $(1,914) 8.85 \pm 0.79$ & 1.00 & Important \\
\hline Serum K & $(4,392) 4.11 \pm 0.86$ & (815) $4.29 \pm 0.64$ & $(3,577) 4.07 \pm 0.89$ & 1.00 & Important \\
\hline Serum Na & $(4,395) 138.18 \pm 4.49$ & (815) $138.79 \pm 5.47$ & $(3,580) 138.05 \pm 4.22$ & 1.00 & Important \\
\hline \multicolumn{6}{|l|}{ Other tests } \\
\hline Vitamin D3 & (220) $31.56 \pm 21.08$ & (59) $35.68 \pm 27.24$ & (161) $30.06 \pm 18.19$ & 0.913 & Marginal \\
\hline D-Dimer & (466) $169.18 \pm 3214.80$ & (158) $6.40 \pm 3377.46$ & $(308) 1729.43 \pm 3041.07$ & 1.00 & Important \\
\hline CRP & $(2,357) 48.66 \pm 45.92$ & $(484) 65.80 \pm 52.98$ & $(1,873) 44.23 \pm 42.83$ & 1.00 & Important \\
\hline Creatinine & $(4,464) 1.54 \pm 4.90$ & (820) $2.11 \pm 1.81$ & $(3,644) 1.41 \pm 5.35$ & 0.99 & Important \\
\hline FBS & $(1,127) 142.69 \pm 62.44$ & (316) $60.92 \pm 72.76$ & (811) $135.59 \pm 56.40$ & 1.00 & Important \\
\hline Blood PH & (552) $7.39 \pm 0.18$ & (150) $7.35 \pm 0.12$ & (402) $7.40 \pm 0.20$ & 1.00 & Important \\
\hline $\mathrm{HCO} 3$ & (552) $27.59 \pm 4.79$ & (150) $25.71 \pm 5.23$ & (402) $28.29 \pm 4.41$ & 1.00 & Important \\
\hline $\mathrm{BE}$ & $(42)-2.44 \pm 2.97$ & (5) $-3.27 \pm 3.86$ & $(37)-2.33 \pm 2.88$ & 1.00 & Important \\
\hline \multicolumn{6}{|l|}{ Interventions } \\
\hline Ventilation & $(4,542) 0.30 \pm 2.59$ & (822) $1.4927 \pm 5.86$ & $(3,720) 0.03 \pm 0.49$ & 1.00 & Important \\
\hline Oxygen-therapy & $(4,542) 0.59 \pm 1.54$ & (822) $0.82 \pm 2.01$ & $(3,720) 0.54 \pm 1.41$ & 1.00 & Important \\
\hline
\end{tabular}

MCV: Mean corpuscular volume; MCH: Mean corpuscular hemoglobin; MCHC: Mean corpuscular hemoglobin concentration; MIX: Ratio of eosinophil, basophil, and monocyte to total white blood cells; WBC: White blood cells; RBC: Red blood cell; RDW-CV: Red cell distribution width-coefficient of variation; PDW: Platelet distribution width; MPV: Mean platelet volume; AST: Aspartate transaminase; ALT: Alanine transaminase; ALP: Alkaline phosphatase; CPK: Creatine phosphokinase; CKMB: Creatine kinase myocardial band; CRP: C-reactive protein; FBS: Fasting blood sugar; $\mathrm{HCO}_{3}$ : Bicarbonate; $\mathrm{BE}$ : Base excess 
4.2. Step one results: Identification of COVID-19 significant risk factors

In this step, the SPSS Modeler ignored predictors with a lot of missing values. Therefore, the number of missing values in the datasets decreased to less than $15 \%$. Accuracy and area under the curve (AUC) index of the testing dataset, as fitness criteria, were $85.87 \%$ and 85.00, respectively. Consequently, the model was fitted well, without overfitting problems (9).

As shown in Table 3 and based on the MLR model, age, ventilation, oxygen therapy, CRP, AST, ALT, ALP, CPK, serum calcium, serum sodium, serum potassium, and creatinine have a significant relationship with the survival of COVID-19 patients.

According to the results, the group in which ventilation was required had a higher MR (Beta=0.81, Sig:0.00). Out of the 222 patients that received this intervention, only 37 (16.67\%) subjects survived. Regarding oxygen therapy, the same results were obtained, with a weaker correlation (Beta $=-0.08$, Sig:0.00). Regarding laboratory test predictors, the results were as follows:

In the current study based on the results of MLR model for surviving patients, mortality showed negative association with the levels of CRP (Beta=0.26, Sig:0.00), AST (Beta $=-0.62$, Sig:0.00), ALP (Beta $=-0.24$, Sig:0.00), CPK (Beta=-0.24, Sig:0.00), sodium (Beta $=-0.20$, Sig:0.00), potassium (Beta=0.14, Sig:0.02), and creatinine (Beta=-0.08, Sig:0.02). Accordingly, it was revealed that mortality had a positive relationship with ALT (Beta=0.21, Sig:0.00) and calcium (Beta=0.32, Sig:0.00).

\subsection{Step two results: Identifying odds ratio of COVID- 19 risk factors}

In the following stage, significant predictors were altered into categorical variables to enter them in the ULR and MLR models and calculate the OR. Thresholds used for the categorization of these predictors were their standard ranges. This transformation enabled us to identify the critical values of laboratory tests, attributed to the patients' survival status (See appendix 1).

According to the ULR results, the age-related OR increased in older ages. The results showed that age groups of 40-49, 50-59, 60-69, 70-79, and above of 80 were associated with elevated crude OR levels of 1.86 (CI: 1.14-3.03), 3.38 (CI: 2.19-5.22), 5.07 (CI: 3.317.76), 9.24 (CI: 6.09-14.02), and 14.31 (CI: 9.3821.84) respectively. Therefore, age was found to be an essential COVID-19 risk factor that should not be overlooked. Other significant risk factors were related to liver injury, including ALT higher than the reference range of $55-100(\mathrm{OR}=2.27, \mathrm{CI}: 1.73-2.97)$ and above of $100(\mathrm{OR}=3.94, \mathrm{CI}: 2.86-5.44)$, AST range of 40-100 IU/L (OR=2.69, CI: 2.19-3.30) and above of $100 \mathrm{IU} / \mathrm{L}(\mathrm{OR}=6.05, \mathrm{CI}: 4.85-7.54)$, and ALP higher than $200 \mathrm{IU} / \mathrm{L}(\mathrm{OR}=2.81, \mathrm{CI}: 2.04-3.88)$. Serum electrolytes, including sodium under $135 \mathrm{mEq} / \mathrm{l}$ $(\mathrm{OR}=1.79$, CI: 1.43-2.24), and over $145 \mathrm{mEq} / \mathrm{l}$ $(\mathrm{OR}=7.22$, CI: $5.03-10.36)$, potassium above of 5.50 $\mathrm{mEq} / \mathrm{l}(\mathrm{OR}=6.57, \mathrm{CI}: 3.73-11.55)$, and calcium under $8.50 \mathrm{mEq} / \mathrm{l}(\mathrm{OR}=3.44, \mathrm{CI}: 2.85-4.16)$ were related to increased MR due to COVID-19. Furthermore, elevated CPK ranges of 307-600 and 192-400 IU/L (male and female, respectively) (OR=2.81, CI: 2.18 3.63) and above of 600 and $400 \mathrm{IU} / \mathrm{L}$ (male and female, respectively) (OR=4.09, CI: 3.15-5.32), CRP more than $100 \mathrm{mg} / \mathrm{l} \quad(\mathrm{OR}=2.68, \mathrm{CI}: 1.70-4.23)$, and creatinine more than $1.5 \mathrm{mg} / \mathrm{l}(\mathrm{OR}=6.85, \mathrm{CI}: 5.69$ 8.25) were associated with COVID-19 MR. More information about crude and adjusted OR are presented in Table 4.

4.4. Step three results: Identifying high-risk patients using Chi-square automatic interaction detector model

Based on the previous analyses, 9 out of 33 analyzed laboratory test parameters had a significant

Table 3. Multiple logistic regression results; target variable is the outcome (survived or deceased)

\begin{tabular}{|c|c|c|c|c|c|c|c|}
\hline \multirow{3}{*}{$\begin{array}{l}\text { Group } \\
\text { Survived }\end{array}$} & \multicolumn{7}{|c|}{ Parameter Estimates } \\
\hline & & Beta coefficient & Std. Error & P-value & $\operatorname{Exp}(B)$ & \multicolumn{2}{|c|}{ 95\% Confidence Interval for $\operatorname{Exp}(\mathrm{B})$} \\
\hline & & & & & & Lower bound & Upper bound \\
\hline & Intercept & 4.87 & & 0.00 & 115.58 & & \\
\hline & Age & -0.05 & 0.00 & 0.00 & 0.95 & 0.95 & 0.96 \\
\hline & [Gender=1.000] & -0.01 & 0.11 & 0.93 & 0.99 & 0.81 & 1.22 \\
\hline & [Gender $=2.000]$ & $0^{\mathrm{b}}$ & & & & & \\
\hline & Ventilation & -0.81 & 0.08 & 0.00 & 0.45 & 0.38 & 0.52 \\
\hline & Oxygen therapy & -0.08 & 0.03 & 0.00 & 0.93 & 0.88 & 0.98 \\
\hline & CRP & -0.26 & 0.05 & 0.00 & 0.77 & 0.71 & 0.84 \\
\hline & Creatinine & -0.08 & 0.03 & 0.02 & 0.92 & 0.87 & 0.99 \\
\hline & AST & -0.62 & 0.09 & 0.00 & 0.54 & 0.45 & 0.64 \\
\hline & ALT & 0.21 & 0.07 & 0.00 & 1.23 & 1.07 & 1.42 \\
\hline & ALP & -0.24 & 0.05 & 0.00 & 0.79 & 0.71 & 0.87 \\
\hline & $\mathrm{CPK}$ & -0.24 & 0.05 & 0.00 & 0.78 & 0.71 & 0.86 \\
\hline & Serum calcium & 0.32 & 0.06 & 0.00 & 1.38 & 1.24 & 1.54 \\
\hline & Serum potassium & -0.14 & 0.06 & 0.02 & 0.87 & 0.78 & 0.98 \\
\hline & Serum sodium & -0.20 & 0.06 & 0.00 & 0.82 & 0.74 & 0.91 \\
\hline
\end{tabular}

a: The reference category is: dead.

b: This parameter is set to zero since it is redundant.

CRP: C-reactive protein; AST: Aspartate transaminase; ALT: Alanine transaminase; ALP: Alkaline phosphatase; CPK: Creatine phosphokinase 
relationship with the patient's survival rate. These nine parameters with categorical amounts were entered into the CHAID node to develop a decision tree model.

According to the CHAID model, the patients were classified into 5 categories based on their risk factors, namely low risk (under $20 \% \mathrm{MR}$ ), moderate risk 1 (20-30\% MR), moderate risk 2 (30-40\% MR), high risk (40-50\% MR), and very high risk (more than $50 \% \mathrm{MR}$ ). These risk factors were separately defined for each age group (Figure 1). In the ages under 40, sodium, CPK, AST, and creatinine (accuracy $=96.25 \%, A U C=0.63$ ), in the age group of 40-59, AST, calcium, creatinine, and sodium (accuracy $=88.57 \%, \mathrm{AUC}=0.75$ ), within 60-69-yearold patients, AST, creatinine, CPK, ALP, and CRP (accuracy $=82.70 \%, \mathrm{AUC}=0.71$ ), in $70-79$ age group,
AST, creatinine, calcium, ALP, and CPK (accuracy= $8.00 \%, A U C=0.83$ ), and for those above 80 , creatinine, AST and sodium (accuracy $=62.16 \%, \mathrm{AUC}=0.63$ ) were associated with high-risk COVID-19 patients.

In the age under 40 years (4.64\% MR), with normal creatinine, an increase in AST of $\geq 100 \mathrm{IU} / \mathrm{L}$, with CPK of $\geq 307$ IU/L in male patients and CPK of $\geq$ 192 IU/L in female patients were associated with $41.67 \%$ MR. Therefore, this age group was considered high-risk patients. Moreover, in this age group, $75.00 \%$ of MR correlated with elevated creatinine levels of $\geq 1.5 \mathrm{mg} / \mathrm{l}$ and an abnormal sodium level.

In the age group of 40-59 years (11.84\% MR), high-risk group patients have been seen as, as follows:

Patients with creatinine levels of $\geq 1.5 \mathrm{mg} / \mathrm{l}$ that

\begin{tabular}{|c|c|c|c|c|}
\hline \multirow{2}{*}{ Variables } & \multirow{2}{*}{$\begin{array}{c}\text { Crude } \\
\text { OR }(95 \% \mathrm{CI}) \\
\end{array}$} & \multicolumn{3}{|c|}{ Adjusted } \\
\hline & & P-value & OR (95\% CI) & P-value \\
\hline \multicolumn{5}{|l|}{ Age (years) } \\
\hline Under 40 & 1 & & 1 & \\
\hline $40-49$ & 1.86 (CI: 1.14-3.03) & 0.01 & 1.62 (CI: 0.96-2.75) & 0.07 \\
\hline $50-59$ & 3.38 (CI: $2.19-5.22$ ) & 0.00 & 2.42 (CI: $1.51-3.88)$ & 0.00 \\
\hline $60-69$ & 5.07 (CI: 3.31-7.76) & 0.00 & 3.48 (CI: $2.19-5.53$ ) & 0.00 \\
\hline $70-79$ & 9.24 (CI: $6.09-14.02)$ & 0.00 & 5.22 (CI: $3.30-8.23$ ) & 0.00 \\
\hline Above 80 & 14.31 (CI: 9.38-21.84) & 0.00 & 7.05 (CI: 4.44-11.21) & 0.00 \\
\hline \multicolumn{5}{|l|}{ Gender } \\
\hline Male & 1.14 (CI: 0.96-1.36) & 0.13 & 0.98 (CI: 0.80-1.21) & 0.88 \\
\hline Female & 1 & & 1 & \\
\hline \multicolumn{5}{|l|}{ ALT (IU/L) } \\
\hline Up to 55 & 1 & & 1 & \\
\hline $55-100$ & 2.27 (CI: 1.73-2.97) & 0.00 & 1.12 (CI: 0.78-1.59) & 0.53 \\
\hline Above 100 & 3.94 (CI: 2.86-5.44) & 0.00 & 1.12 (CI: 0.72-1.73) & 0.61 \\
\hline \multicolumn{5}{|l|}{ AST (IU/L) } \\
\hline Up to 40 & 1 & & 1 & \\
\hline $40-100$ & 2.69 (CI: 2.19-3.30) & 0.00 & 1.64 (CI: $1.27-2.11)$ & 0.00 \\
\hline Above 100 & 6.05 (CI: 4.85-7.54) & 0.00 & 3.21 (CI: $2.42-4.25)$ & 0.00 \\
\hline \multicolumn{5}{|l|}{ ALP (IU/L) } \\
\hline $40-129$ & 1 & & 1 & \\
\hline $129-200$ & 1.02 (CI: 0.75-1.39) & 0.89 & 1.33 (CI: 0.92-1.92) & 0.13 \\
\hline Above 200 & 2.81 (CI: 2.04-3.88) & 0.00 & 2.17 (CI: $1.47-3.20)$ & 0.00 \\
\hline \multicolumn{5}{|l|}{ CPK (IU/L) } \\
\hline Under 39 (male), under 26 (female) & 0.43 (CI: 0.22-1.39) & 0.10 & 0.24 (CI: 0.08-0.74) & 0.01 \\
\hline 39-307 (male), 26-191 (female) & 1 & & 1 & \\
\hline 307-600 (male), 192-400 (female) & 2.81 (CI: 2.18-3.63) & 0.00 & 2.24 (CI: $1.65-3.04)$ & 0.00 \\
\hline Above 600 (male), Above 400 (female) & 4.09 (CI: 3.15-5.32) & 0.00 & 2.21 (CI: 1.58-3.08) & 0.00 \\
\hline \multicolumn{5}{|l|}{ Serum $\mathrm{Na}(\mathrm{mEq} / \mathrm{l})$} \\
\hline Under 135 & 1.79 (CI: $1.43-2.24)$ & 0.00 & $1.31(\mathrm{CI}: 1.01-1.70)$ & 0.04 \\
\hline $135-145$ & 1 & & 1 & \\
\hline Above 145 & 7.22 (CI: $5.03-10.36)$ & 0.00 & 5.05 (CI: 3.20-7.96) & 0.00 \\
\hline \multicolumn{5}{|l|}{ Serum Ca (mEq/l) } \\
\hline Under 8.50 & 3.44 (CI: $2.85-4.16)$ & 0.00 & 2.31 (CI: 1.85-2.91) & 0.00 \\
\hline Above 8.50 & 1 & & 1 & \\
\hline Serum K (mEq/l) & 0.83 (CI: 0.57-1.21) & 0.34 & 0.85 (CI: 0.54-1.31) & 0.46 \\
\hline Under 3.50 & $\begin{array}{c}0.83(\text { Cl: } 0.5 /-1.21) \\
1\end{array}$ & 0.34 & $\begin{array}{c}0.85 \text { (LI: } 0.54-1.31 \text { ) } \\
1\end{array}$ & 0.40 \\
\hline $\begin{array}{l}3.50-5.49 \\
\text { Above } 5.50\end{array}$ & 6.57 (CI: 3.73-11.55) & 0.00 & 2.41 (CI: 1.21-4.77) & 0.01 \\
\hline \multicolumn{5}{|l|}{ Creatinine (mg/l) } \\
\hline Under 1.5 & 1 & & 1 & \\
\hline Above 1.5 & 6.85 (CI: 5.69-8.25) & 0.00 & 3.43 (CI: 2.76-4.27) & 0.00 \\
\hline \multicolumn{5}{|l|}{ CRP (mg/l) } \\
\hline Under 8 & 1 & & 1 & \\
\hline $8-100$ & 1.15 (CI: 0.80-1.65) & 0.45 & 0.89 (CI: 0.58-1.35) & 0.57 \\
\hline Above 100 & 2.68 (CI: $1.70-4.23)$ & 0.00 & 1.66 (CI: $0.97-2.86)$ & 0.06 \\
\hline
\end{tabular}

CRP: C-reactive protein; AST: Aspartate transaminase; ALT: Alanine transaminase; ALP: Alkaline phosphatase; CPK: Creatine phosphokinase 


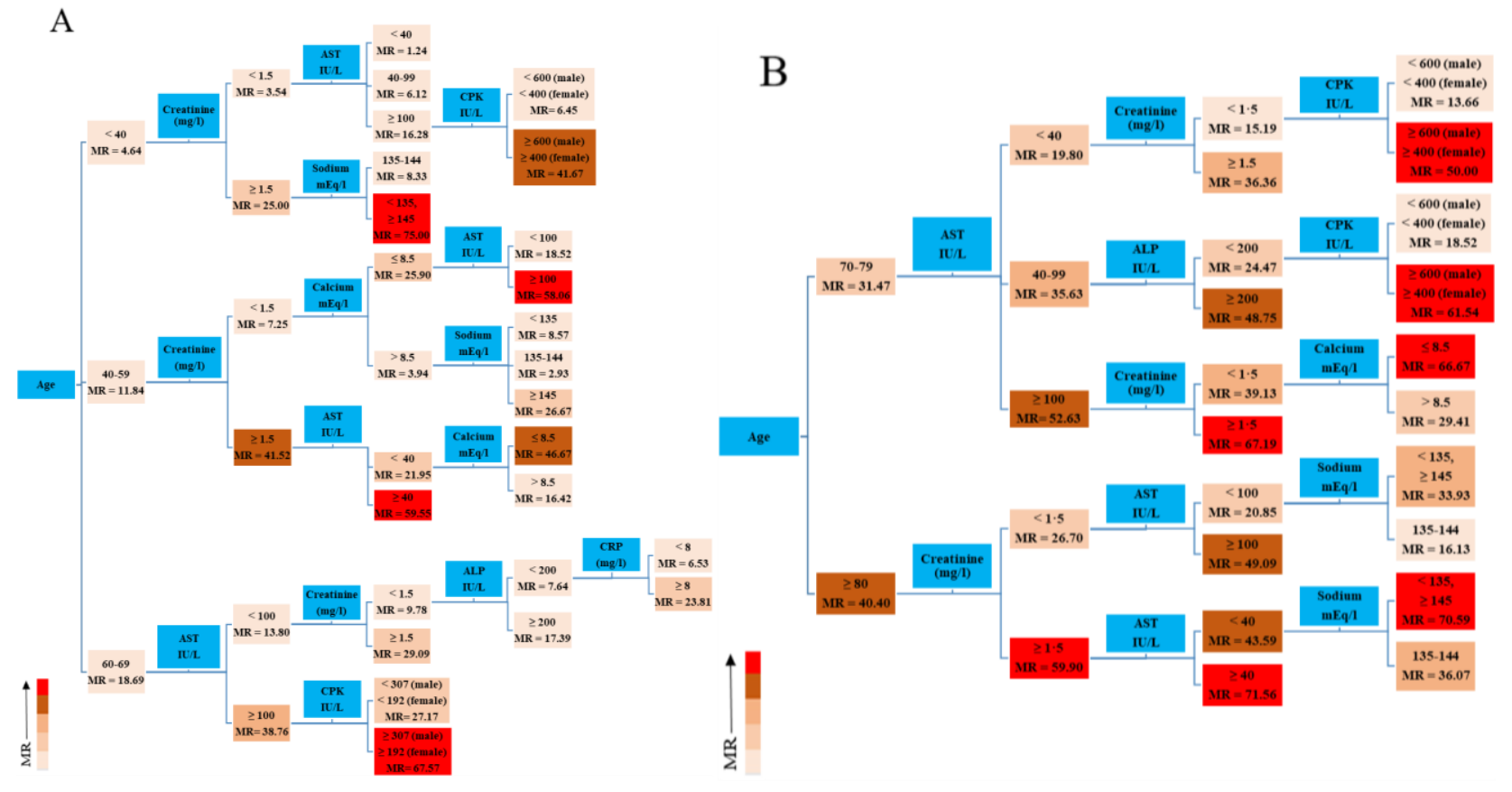

Figure 1. Results of decision tree model (Chi-square automatic interaction detector model) in patients under 70 (A), and over 70 (B)

have $41.52 \% \mathrm{MR}$, patients with hypocalcemia, and AST of $\geq 100 \mathrm{IU} / \mathrm{L}$ that have $56.08 \% \mathrm{MR}$, patients with creatinine levels of $\geq 1.5 \mathrm{mg} / \mathrm{l}$ and hypocalcemia that have $46.67 \% \mathrm{MR}$, and patients with creatinine levels of $\geq 1.5 \mathrm{mg} / \mathrm{l}$ and AST of $\geq 40 \mathrm{IU} / \mathrm{L}$ that have $59.55 \% \mathrm{MR}$, hence regarded as high-risk COVID-19 patients.

In the age group of $60-69$ years (18.69\% MR), elevated AST of $\geq 100 \mathrm{IU} / \mathrm{L}$ with $\mathrm{CPK}$ of $\geq 307 \mathrm{IU} / \mathrm{L}$ in male patients and CPK of $\geq 192 \mathrm{IU} / \mathrm{L}$ in female patients were associated with $67.57 \% \mathrm{MR}$.

In the age group of $70-79$ (31.47\% MR), the six groups of high-risk patients were as follows:

Male patients with CPK of $\geq 307$ IU/L and female patients with CPK of $\geq 192 \mathrm{IU} / \mathrm{L}$ had $5.00 \%$ MR. In patients with AST level of 40-99 IU/L and elevated ALP of $\geq 200$ IU/L and those with elevated CPK of $\geq$ $600 \mathrm{IU} / \mathrm{L}$ (male) and $\mathrm{CPK}$ of $\geq 400 \mathrm{IU} / \mathrm{L}$ (female), MRs were 48.75\% and 61.54\%, respectively. Furthermore, in this age group, there was $52.63 \%$ MR with AST of $\geq 100 \mathrm{IU} / \mathrm{L}, 67.19 \%$ MR with AST of $\geq 100 \mathrm{IU} / \mathrm{L}$, and creatinine level of $\geq 1.5 \mathrm{mg} / \mathrm{l}$, and $66.67 \%$ MR with AST of $\geq 100 \mathrm{IU} / \mathrm{L}$, and there was also hypocalcemia.

In the age above 80 years (4.40\% MR), due to COVID-19, MRs were 49.09\%, 59.90\%, and 79.59\% with AST of $\geq 100 \mathrm{IU} / \mathrm{L}$, creatinine of $\geq 1.5 \mathrm{mg} / \mathrm{l}$ (43.59\% when AST of $<40$ IU/L and $71.56 \%$ when AST of $\geq 40 \mathrm{IU} / \mathrm{L}$ ), and creatinine level of $\geq 1.5 \mathrm{mg} / \mathrm{l}$, respectively, and there were also abnormal sodium levels. More information is presented in Figure 1 (For further details, see Appendix 2).

\section{Discussion}

According to the results of MLR and ULR analysis, AST, ALP, ALT, calcium, sodium, potassium, creatinine, CPK, and CRP were correlated with the risk of death in COVID-19 patients. Furthermore, in the decision tree model, high-risk patients were identified.

\subsection{Age}

In this study, a strong relationship was found between age and COVID-19 MR. The results of the present research confirmed those of the previous studies indicating that older age was an important risk factor of death in patients with COVID-19 (10, 11). It can be assumed that age-dependent defects in humoral and cellular immune function could lead to a decrease in the number of T cells, phagocytosis, and levels of interferon (12).

\subsection{Liver enzymes}

According to the results, elevated AST, ALT, and ALP above the normal range were associated with an increased risk of death in COVID-19 patients in all age groups. Liver injury, and subsequently abnormal levels of AST, ALT, and ALP, are prevalent in patients with COVID-19 during disease progression and longer hospital stays $(13,14)$. A serum marker of mitochondrial damage in hepatocytes is high levels of AST. In severe cases of patients with COVID-19, liver damage is associated with liver hypoxia/ reperfusion injury, which in turn leads to 
mitochondrial apoptosis, triggers cell injury and necrosis, and finally elevates AST levels (15). Abnormal levels of liver enzymes can be caused by viral infections, such as COVID-19 (16), hepatotoxic drug consumption, or by an immune-mediated inflammatory response during treatment. For this reason, more intensive care and specific therapeutic approaches are needed for patients with severe COVID-19 who have pre-existing liver diseases and, especially, are older (17).

\subsection{Serum electrolytes}

Abnormal serum electrolytes (calcium, potassium, and sodium) were also among the risk factors associated with the MR of COVID-19. Concerning serum calcium, the results of the current study were consistent with those of previous ones regarding COVID-19, which suggested that patients with hypocalcemia had more severe symptoms and MRs than others (18). This finding is attributable to the role of calcium in immune response development (19). Hypocalcemia is associated with a higher incidence of organ injury, hyperproteinemia, unbalanced vitamin $\mathrm{D}$, and parathyroid hormone. Therefore, the early detection and correction of $\mathrm{Ca}$ levels in patients with hypocalcemia may lead to better outcomes and a reduction in the severity of symptoms (20).

Moreover, the results showed that both hyponatremia and hypernatremia were correlated with high COVID-19 MR. Lippi et al. reported that in patients with COVID-19, serum electrolytes levels, including sodium, were decreased (21). In viral infections, low sodium levels may cause tissue damage and increase the severity of the viral disease (22). The results of previous studies showed that sodium was an essential factor regulating the expression of angiotensin-converting enzyme-2 (ACE2) in the body. Additionally, the COVID-19 virus enters the host cell by connecting to this receptor, and hyponatremia may cause an overexpression of the ACE2 receptor in the long term. Consequently, hyponatremia may generate more severe conditions of COVID-19 (23). In addition, one of the clinical symptoms of COVID-19 is diarrhea and water loss, (24) which can cause hypernatremia in the body (25). It has been seen that hypernatremia and hyponatremia have some adverse effects on various organ functions, such as the central nervous system and, in turn, an increased MR (26).

High-level serum potassium is another risk factor that is found to be associated with COVD-19 MR. Hyperkalemia may be a result of other underlying diseases, such as kidney dysfunction, and can lead to cardiac arrest. Evidence also suggests that immunodeficiency against viral infections could lead to hyperkalemia (27). However, hyperkalemia, for whatever reason, is dangerous in itself and must be carefully monitored.

\subsection{Creatine phosphokinase}

Elevated CPK is another risk factor that is shown to be associated with a high MR in patients. Increased $\mathrm{CPK}$ is caused by damage to muscles (mainly skeletal muscles) (28). Based on the findings of previous studies, patients with influenza type A have increased CPK levels due to skeletal muscle involvement (29). Furthermore, it has been reported that rhabdomyolysis and elevated $\mathrm{CPK}$ can occur in COVID-19 patients (30). The mechanism of COVID19-induced rhabdomyolysis, however, has not yet been understood (31). The only known fact is that elevated CPK of $\geq 150 \mathrm{IU} / \mathrm{L}$ is a common finding in patients with COVID-19 (32). Therefore, increased $\mathrm{CPK}$, which may be due to muscle involvement in the severe stages of the disease, becomes a predictor for higher MR in patients with COVID-19.

\subsection{C-reactive protein}

Finally, the results showed that elevated CRP of above $8 \mathrm{mg} / \mathrm{dl}$ was associated with an increase in COVID-19 MR. In this respect, and in line with the results of previous studies, elevated CRP, as an inflammatory biomarker, could indicate lung damage, and therefore, represent the severity of the respiratory tract involvement in patients with COVID19 (33).

\subsection{Creatinine}

In line with recent reports, the results of this study indicated that an elevated level of creatinine in patients with COVID-19 was observed and associated with MR. These results demonstrated that creatinine levels can be a predictor of renal impairment, one of the most prominent causes of death among patients. Furthermore, Cheng et al. reported that a high level of creatinine increases the probability of admission to the intensive care unit, and these patients have a higher risk of deterioration (34). Angiotensinconverting enzyme- 2 receptors expression in human kidneys and bladder could be a potential binding route of coronavirus (35). Since researchers have isolated coronavirus from urine samples of some patients, the association of kidney impairment with COVID-19 seemed to be possible (36).

In this study, the risk factors of COVID-19 were analyzed through logistic regression and final predictors were identified displaying robust results. According to the results, abnormal levels of some laboratory tests appeared in patients with COVID-19, which can be due to non-respiratory multi-organ involvements seen during the course of COVID-19. These include the liver, heart, gastrointestinal tract, and kidney (14). These abnormalities are not specific to the novel coronavirus infection (36); nevertheless, prompt action for their management as potential risk factors for COVID-19 severity may reduce its morbidity and mortality rates.

Considering the fact that there is currently no 
approved treatment for COVID-19, our findings support the idea that in patients with COVID-19, identifying high-risk patients and providing supportive care to prevent organ damage and homeostasis abnormalities have a crucial role in the outcome of the therapeutic interventions.

\section{Limitations}

As a retrospective cohort study, this research had some inherent limitations. Some patients had incomplete data in their medical records. Moreover, slightly different in-hospital procedures led to a lack of order or records of some laboratory tests. A large sample size of the study, however, may overcome these drawbacks.

\section{Acknowledgments}

The authors would like to thank Mohammad Hossein Shoushatri for his guidance in data mining and comments that greatly improved the manuscript. They would also like to acknowledge Mahtab Moradi and Elham Salehian for their help with data acquisition. The authors would also like to express their gratitude to Sajad Kamali for data preparation and to Zahra Ghalkhani for her expert advice on laboratory sciences.

The authors of this manuscript, who meet the requirements for authorship that previously explained, declare that they have read and accepted this manuscript. They also approve the honesty and correctness of this work.

\section{Footnotes}

Author's contributions: A.M. contributed to the conception and design of the study, analysis, and drafting of the article. S.R. contributed to the analysis, interpretation, and drafting of the article. J.S. and A.Mi. contributed to the interpretation and critical revision of the article. F.P. contributed to data acquisition and provided a critical revision of the article. N.Y. contributed to the conception, data acquisition, and provided critical revision of the article.

Conflicts of Interest: The authors declare that there is no conflict of interest.

Ethical Approval: This study was approved by the Ethics Committee of the Shahid Beheshti University of Medical Sciences, Tehran, Iran, (IR.SBMU. RETECH.REC.1399.007).

Funding/Support: This research was funded by the Vise Chancellor in Research Affairs of the Shahid Beheshti University of Medical Science under contract number 99-22935.

Informed Consent: Not applicable.

\section{References}

1. Lillie PJ, Samson A, Li A, Adams K, Capstick R, Barlow GD, et al.
Novel coronavirus disease (Covid-19): The first two patients in the UK with person to person transmission. J Infect. 2020; 80(5):578-606. doi: 10.1016/j.jinf.2020.02.020. [PubMed: 32119884].

2. Chen G, Wu D, Guo W, Cao Y, Huang D, Wang H, et al. Clinical and immunological features of severe and moderate coronavirus disease 2019. J Clin Invest. 2020;130(5):2620-9. doi: 10.1172/JCI137244. [PubMed: 32217835].

3. Shirani K, Toghyani A. COVID-19 pneumonia with scant respiratory symptoms. J Res Med Sci. 2020;25:82. doi: 10.4103/jrms.JRMS_326_20. [PubMed: 33088319].

4. Takian A, Raoofi A, Kazempour-Ardebili S. COVID-19 battle during the toughest sanctions against Iran. Lancet. 2020; 395(10229):1035-6. doi: 10.1016/S0140-6736(20)30668-1. [PubMed: 32199073].

5. Castro MC, de Carvalho LR, Chin T, Kahn R, Franca GVA, Macario EM, et al. Demand for hospitalization services for COVID-19 patients in Brazil. MedRxiv. 2020;3:1-16. doi: 10.1101/2020.03.30.20047662.

6. Kuo CY, Yu LC, Chen HC, Chan CL. Comparison of models for the prediction of medical costs of spinal fusion in Taiwan diagnosisrelated groups by machine learning algorithms. Healthc Inform Res. 2018;24(1):29-37. doi: 10.4258/hir.2018.24.1.29. [PubMed: 29503750].

7. Maniruzzaman M, Rahman MJ, Ahammed B, Abedin MM. Classification and prediction of diabetes disease using machine learning paradigm. Heal Inf Sci Syst. 2020;8(1):7. doi: 10.1007/s13755-019-0095-z. [PubMed: 31949894].

8. Hersh AL, Black WC, Tosteson ANA. Estimating the population impact of an intervention: a decision-analytic approach. Stat Methods Med Res. 1999;8(4):311-30. doi: 10.1177/09622802 9900800404. [PubMed: 10730336].

9. McCormick K, Abbott D. IBM SPSS modeler cookbook. Birmingham: Packt Publ; 2020.

10. Zandkarimi E, Moradi G, Mohsenpour B. The prognostic factors affecting the survival of Kurdistan province COVID-19 patients: a cross-sectional study from February to May 2020. Int J Heal Policy Manag. 2020;In Press. doi: 10.34172/ijhpm.2020.155. [PubMed: 32861230].

11. Sun H, Ning R, Tao Y, Yu C, Deng X, Zhao C, et al. Risk factors for mortality in 244 older adults with COVID-19 in Wuhan, China: a retrospective study. Am Geriatr Soc. 2020;6(6):E19-23. doi: 10.1111/jgs.16533. [PubMed: 32383809].

12. Sharma G, Goodwin J. Effect of aging on respiratory system physiology and immunology. Clin Interv Aging. 2006;1(3):25360. doi: 10.2147/ciia.2006.1.3.253. [PubMed: 18046878].

13. Fan Z, Chen L, Li J, Cheng X, Yang J, Tian C, et al. Clinical features of COVID-19-related liver functional abnormality. Clin Gastroenterol Hepatol. 2020;18(7):1561-6. doi: 10.1016/j.cgh. 2020.04.002. [PubMed: 32283325].

14. Chen N, Zhou M, Dong X, Qu J, Gong F, Han Y, et al. Epidemiological and clinical characteristics of 99 cases of 2019 novel coronavirus pneumonia in Wuhan, China: a descriptive study. Lancet. 2020;395(10223):507-13. doi: 10.1016/S01406736(20)30211-7. [PubMed: 32007143].

15. Cannistrà $M$, Ruggiero $M$, Zullo $A$, Gallelli $G$, Serafini $S$, Maria $M$, et al. Hepatic ischemia reperfusion injury: A systematic review of literature and the role of current drugs and biomarkers. Int J Surg. 2016;33(Suppl 1):S57-70. doi: 10.1016/j.ijsu.2016.05.050. [PubMed: 27255130].

16. Xu L, Liu J, Lu M, Yang D, Zheng X. Liver injury during highly pathogenic human coronavirus infections. Liver Int. 2020; 40(5):998-1004. doi: 10.1111/liv.14435. [PubMed: 32170806].

17. Zhang C, Shi L, Wang FS. Liver injury in COVID-19: management and challenges. Lancet Gastroenterol Hepatol. 2020;5(5):428-30. doi: 10.1016/S2468-1253(20)30057-1. [PubMed: 32145190].

18. Sun JK, Zhang WH, Zou L, Liu Y, Li JJ, Kan XH, et al. Serum calcium as a biomarker of clinical severity and prognosis in patients with coronavirus disease 2019: a retrospective crosssectional study. Crit Care Emerg Med. 2020;12(12):11287-95. doi: 10.18632/aging.103526. [PubMed: 32589164].

19. Grinstein S, Klip A. Calcium homeostasis and the activation of calcium channels in cells of the immune system. Bull N Y Acad 
Med. 1989;65(1):69-79. [PubMed: 2557949].

20. El-kurdi B, Khatua B, Rood C, Snozek C, Cartin-Ceba R, Singh VP, et al. Mortality from covid-19 increases with unsaturated fat, and may be reduced by early calcium and albumin supplementation. Gastroenterology. 2020;159(3):1015-8. doi: 10.1053/j.gastro.2020.05.057. [PubMed: 32470338].

21. Lippi G, South AM, Henry BM. Electrolyte imbalances in patients with severe coronavirus disease 2019 (COVID-19). Ann Clin Biochem. 2020;57(3):262-5. doi: 10.1177/0004563220922255. [PubMed: 32266828].

22. Cao YC, Deng QX, Dai SX. Remdesivir for severe acute respiratory syndrome coronavirus 2 causing COVID-19: An evaluation of the evidence. Travel Med Infect Dis. 2020;35:101647. doi: 10.1016/j.tmaid.2020.101647.[PubMed: 32247927].

23. Luo Y, Li Y, Dai J. Low blood sodium increases risk and severity of COVID-19: a systematic review, meta-analysis and retrospective cohort study. MedRxiv. 2020;5:1-24. doi: 10.1101/2020.05.18.20102509.

24. Huang C, Wang Y, Li X, Ren L, Zhao J, Hu Y, et al. Clinical features of patients infected with 2019 novel coronavirus in Wuhan, China. Lancet. 2020;395(10223):497-506. doi: 10.1016/S0140-6736(20)30183-5. [PubMed: 31986264].

25. Drogué HJ, Adias NE. Hypernatremia. $N$ Engl J Med. 2000;342(20):1493-9. doi: 10.1056/NEJM200005183422006. [PubMed: 10816188].

26. Kovesdy CP, Lott EH, Lu JL, Malakauskas SM, Ma JZ, Molnar MZ, et al. Hyponatremia, hypernatremia, and mortality in patients with chronic kidney disease with and without congestive heart failure. Circulation. 2012;125(5):677-84. doi: 10.1161/CIRCUL ATIONAHA.111.065391. [PubMed: 22223429].

27. Parham WA, Mehdirad AA, Biermann KM, Fredman CS. Hyperkalemia revisited. Texas Hear Inst J. 2006;33(1):40-7. [PubMed: 16572868].

28. Do TT. What is the role of creatine phosphokinase (CPK) determination in the evaluation of muscular dystrophy? Medscape. Available at: https://www.medscape.com/answers/ 1259041-96568/what-is-the-role-of-creatine-phosphokinasecpk-determination-in-the-evaluation-of-muscular-dystrophy; 2018.

29. Kessler HA, Trenholme GM, Vogelzang NJ, Semel JD, Harris AA, Levin S, et al. Elevated creatine phosphokinase levels associated with influenza A/Texas/1/77 infection. Scand J Infect Dis. 2015;15(1):7-10. doi: 10.3109/inf.1983.15.issue1.02. [PubMed: 6844879].

30. Suwanwongse K, Shabarek N. Rhabdomyolysis as a presentation of 2019 novel coronavirus disease. Cureus. 2020;12(4):e7561. doi: 10.7759/cureus.7561. [PubMed: 32382463].

31. Ayala E, Kagawa FT, Wehner JH, Tam J, Upadhyay D. Rhabdomyolysis associated with 2009 influenza A(H1N1). JAMA. 2009;302(17):1863-4. doi: 10.1001/jama.2009.1582. [PubMed: 19887664].

32. Laboratory features associated with severe COVID-19. UpToDate. Available at: URL: https://www.uptodate.com/contents/ image/print?imageKey=ID\%2F127820\&topicKey=ID\%2F1274 29\&source=see_link; 2021.

33. Wang L. C-reactive protein levels in the early stage of COVID19. Med Mal Infect. 2020;50(4):332-4. doi: 10.1016/j.medmal. 2020.03.007. [PubMed: 32243911].

34. Cheng Y, Luo R, Wang K, Zhang M, Wang Z, Dong L, et al. Kidney disease is associated with in-hospital death of patients with COVID-19. Kidney Int. 2020;97(5):829-38. doi: 10.1016/ j.kint.2020.03.005. [PubMed: 32247631].

35. Lin W, Hu L, Zhang Y, Ooi JD, Meng T, Jin P, et al. Single-cell analysis of ACE2 expression in human kidneys and bladders reveals a potential route of $2019-\mathrm{nCoV}$ infection. BioRxiv. 2020;8:1-12. doi: 10.1101/2020.02.08.939892.

36. Singhal T. A review of coronavirus disease-2019 (COVID-19). Indian J Pediatr. 2020;87(4):281-6. doi: 10.1007/s12098-02003263-6. 